\title{
Atrial scar on late gadolinium-enhanced imaging to predict electrical reconnection after pulmonary vein isolation for atrial fibrillation
}

\author{
Hubert Cochet ${ }^{*}$, Marjorie Salel, Stephanie Clement-Guinaudeau, Olivier Corneloup, Michel Montaudon, \\ François Laurent
}

From 19th Annual SCMR Scientific Sessions

Los Angeles, CA, USA. 27-30 January 2016

\section{Background}

Atrial fibrillation (AF) recurrences after pulmonary vein isolation (PVI) have been related to transient blocks leading to electrical reconnection. We assessed the accuracy of late gadolinium-enhanced (LGE) CMR in predicting, characterizing and localizing PV reconnection.

\section{Methods}

LGE CMR was performed 3 months after PVI in 24 patients with paroxysmal AF (age $62 \pm 9$ years, 4 women). Imaging was performed 15 min after contrast in transaxial and sagital orientations using an inversion-recovery prepared and respiratory navigated 3D turboFLASH sequence with fat saturation (pixel size $1.25 \times 1.25 \times 2.5 \mathrm{~mm}$ ). Scar burden $(\mathrm{mL})$ was quantified on transaxial images using adaptive thresholding, with a threshold set 3SD above mean blood pool signal. In addition, scar circumferentiality was assessed visually by 2 observers in consensus, potential gaps being distributed on 4 sectors per vein (sup, ant, inf, post). 1 to 3 days after CMR, all patients underwent a systematic electrophysiological study, regardless of potential AF recurrence, in order to assess for PV reconnection. Each reconnection was characterized by high density activation mapping and distributed on the same sectors.

\section{Results}

During the index procedure, complete isolation resistant to adenosine was successfully obtained on all 4 veins in all patients. At 3 months, PV reconnection was detected in $17 / 24$ (71\%) patients, with a mean extent of $1.3 \pm 1.0$ veins and $1.8 \pm 1.6$ sectors per patient. Scar burden on CMR

IHU Liryc - CHU / Université de Bordeaux, Pessac, France was $10.4 \pm 4.5 \mathrm{~mL}$ (range 3.8 to 21.3 ), and scar gaps were present in 16/24(67\%) patients. Global analysis demonstrated a strong relationship between scar burden and PV reconnection: using an optimal cut-off of $11 \mathrm{~mL}$, scar burden predicted PV reconnection with a sensitivity/ specificity of $82 / 100 \%$, and an inverse relationship was found between scar burden and the extent of PV reconnection $(\mathrm{R}=-0.69, \mathrm{p}<0.001)$. In contrast, on regional analysis the relationship between scar gaps and PV reconnection was limited: on a vein-by-vein basis, scar gaps on CMR predicted PV reconnection with a sensitivity/ specificity of $67 / 92 \%$, and on a sector-by-sector basis the agreement between scar gaps on CMR and PV reconnection was only fair $(\mathrm{k}=0.47, \mathrm{p}<0.001)$.

\section{Conclusions}

The assessment of scar circumferentiality around PV ostia on LGE CMR poorly predicts the presence and location of PV reconnections after PVI. However, the global quantification of scar burden relates to the presence and extent of PV reconnection. These results support the use of postablation CMR to identify patients likely to show AF recurrence, but additional research is desirable before considering guiding redo procedures from post-ablation CMR data.

Published: 27 January 2016
doi:10.1186/1532-429X-18-S1-P201

Cite this article as: Cochet et al:: Atrial scar on late gadoliniumenhanced imaging to predict electrical reconnection after pulmonary vein isolation for atrial fibrillation. Journal of Cardiovascular Magnetic Resonance 2016 18(Suppl 1):P201. 


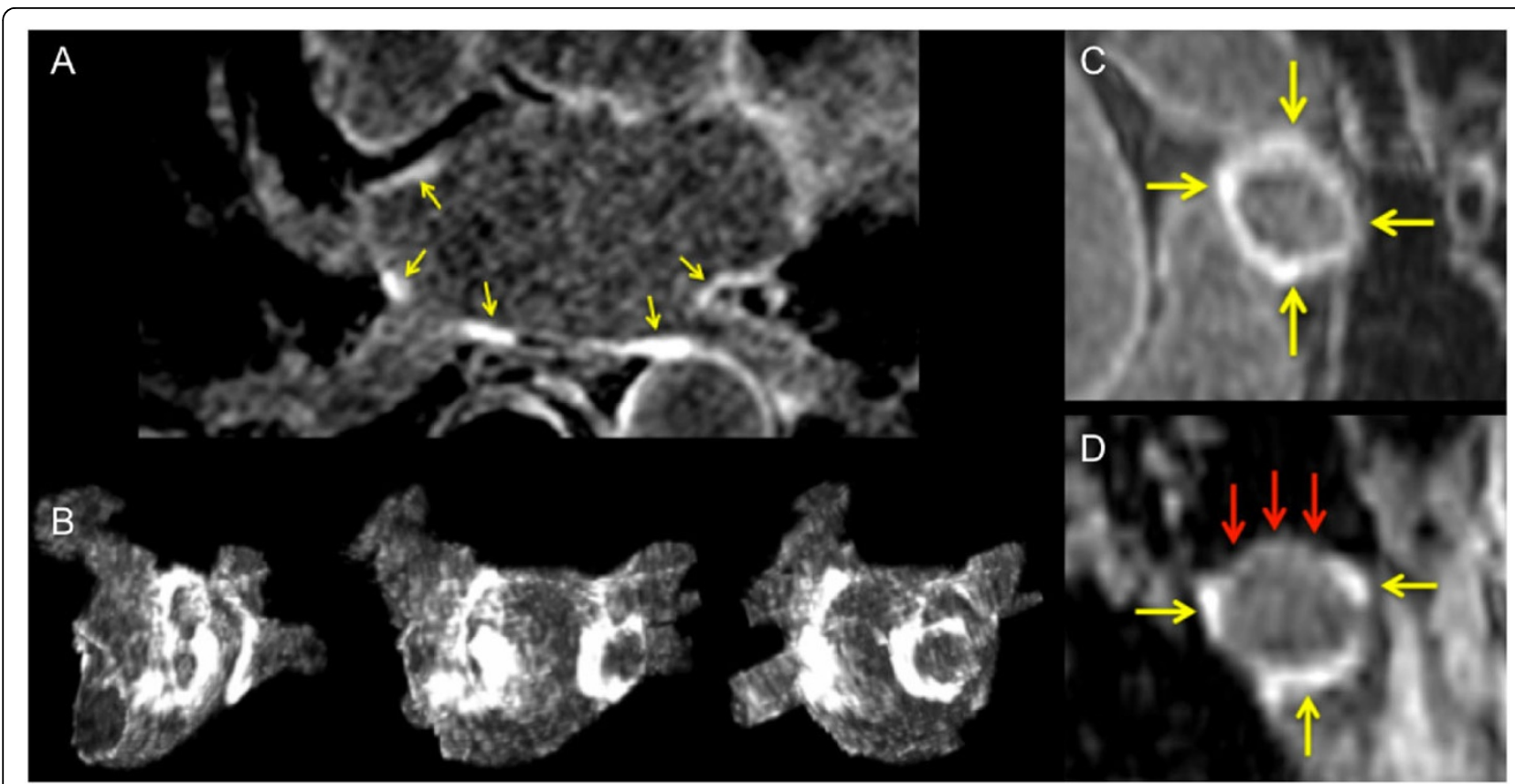

Figure 1 LGE CMR acquired 3 months after pulmonary vein isolation for paroxysmal atrial fibrillation in a 65 year-old man. Transaxial image (A) and maximum intensity projections (B) show scar on the ostium of each pulmonary vein. Scar burden was $15.3 \mathrm{~mL}$. Multiplanar reformat on the ostium of right superior vein (C) shows circumferential scar with no gap (yellow arrows). Multiplanar reformat on right inferior vein (D) shows a scar gap on superior sector (red arrows). Systematic electrophysiological study performed 3 months after the PVI procedure (and 2 days after CMR) demonstrated no pulmonary vein reconnection. 